\title{
Retrospective identification of Acanthamoeba culbertsoni in a case of amoebic meningoencephalitis
}

\author{
E. WILlAERT, A. R. STEVENS, AND G. R. HEALY
}

From the Department of Protozoology, Institute of Tropical Medicine, Antwerp, Belgium; Medical Research Service, Veterans Administration Hospital and Department of Biochemistry and Molecular Biology, University of Florida, Gainesville, Florida, USA; and General Parasitology Branch, Center for Disease Control, Atlanta, Georgia, USA

SUMMARY Acanthamoeba culbertsoni was identified retrospectively in a case of amoebic meningoencephalitis, previously reported by Jager and Stamm (Lancet, 2, 1343, 1972). This is the second report of this species causing secondary infection in man. Positive results were obtained only with anti- $A$. culbertsoni sera when the brain sections were stained by the indirect immunofluorescence antibody test with various antisera produced against different Acanthamoeba species. Antiserum raised against purified plasma membranes of $A$. culbertsoni showed once more its highly specific diagnostic value.

Jager and Stamm (1972) reported the presence of brain abscesses caused by free-living amoebae of the genus Hartmannella (Acanthamoeba) in a patient with Hodgkin's disease. However, these workers were unable to identify specifically the organism that had caused this secondary infection.

We (Willaert and Stevens, 1976) recently reported the identification of Acanthamoeba culbertsoni in a Venezuelan patient with amoebic meningoencephalitis. This was the first report of the identification of this amoeba in a human infection since it was established as an animal pathogen from the work of Culbertson et al. (1959). A. culbertsoni was verified as the infective species in the Venezuelan infection by indirect immunofluorescence, using antiserum that had been produced against purified plasma membranes of $A$. culbertsoni (Stevens et al., 1977). The high specificity of antisera produced in rabbits against purified plasma membranes of different Acanthamoeba species was revealed by various immunological tests. The present report, which concerns the identification of the pathogenic species in the case reported by Jager and Stamm (1972), reemphasises the importance of using antisera produced against cell surface antigens in species identification of acanthamoeba in human infections.

Received for publication 20 December 1977

\section{Material and methods}

\section{PATIENT}

Jager and Stamm (1972) described the case in detail. Briefly, a 27-year-old white American man, who had had Hodgkin's disease for 11 years and who had been treated with ionising radiation and immunosuppressive drugs, was admitted to hospital after having generalised seizures with loss of consciousness. No particular neurological symptoms were observed during the first two days after admission. Thereafter the patient became unresponsive and remained so until death occurred on the fifth day after admission. Necropsy revealed a mild meningitis and brain abscesses, which showed numerous trophozoites and cysts of Hartmannella (Acanthamoeba) sp.

\section{Serological analyses}

Deparaffinised $5-\mu \mathrm{m}$ histological sections of the brain from this patient were stained with haematoxylin and eosin and by the indirect immunofluorescence antibody test. Antisera were produced in rabbits against: (1) whole cells of the species $A$. culbertsoni (A-1), A. astronyxis (Ray), A. polyphaga (P-23), $A$. castellanii (1930), A. royreba (Oak Ridge), A. palestinensis (Reich), A. rhysodes (CCAP-1534), Naegleria fowleri (ITMAP-359), and Entamoeba histolytica (HK-9) (Willaert, 1976); and (2) purified plasma membranes of $A$. castellanii (Neff), $A$. culbertsoni (A-1) (Stevens et al., 1977), and $A$. 
rhysodes (CCAP-1534) (Stevens and Willaert, unpublished results).

The antisera used showed end-point titres from $1: 64$ to $1: 512$ in homologous reaction on in vitro culture antigens. A total absence of cross-reactions with titres as low as 1:16 has been observed in heterologous reaction (Willaert, 1976) except between the antisera of $A$. castellanii, $A$. polyphaga, and $A$. rhysodes, which constitute a group antigenically very closely related. When used on histological sections the antisera stain the antigens up to one dilution below their end-point titres. However, the total absence of cross-reactions allows us to use the antisera at low dilutions, as in the present study, to obtain optimal staining of histological sections.

The patient's serum was also analysed by: (1) indirect immunofluorescence against formalinfixed $A$. castellanii (Neff) and $A$. culbertsoni (A-1); (2) immunoprecipitation against water-soluble antigens of $A$. culbertsoni (A-1), $A$. castellanii (Neff), $A$. rhysodes (CCAP-1534), and $N$. fowleri (Willaert, 1976), or with purified plasma membranes of $A$. culbertsoni (A-1) and $A$. castellanii (Neff) that had been solubilised with Triton-X 100 (Stevens et al., 1977); and (3) agglutination with $A$. culbertsoni (A-1) and $A$. castellanii (Neff). Details of the indirect immunofluorescence and immunoprecipitation techniques have been described previously (Willaert, 1976). Agglutination was performed as outlined by Stevens et al. (1977).

\section{Results}

The histological sections revealed necrotic, haemorrhagic areas in which many amoebae were seen. From the presence of encysted amoebae in the brain sections, which had the typical morphological appearance of Acanthamoeba cysts, the diagnosis on the level of the genus was made. The trophozoites were generally round and had a nucleus with a large, spherical, centrally placed nucleolus surrounded by a hyaline zone. The nucleolus was frequently vacuolated. A nuclear membrane was generally well distinguished, as was the filiformous chromatin substance radiating from the nucleolus towards the nuclear membrane. The cytoplasm usually was highly vacuolated. The trophozoites and cysts averaged $12 \mu \mathrm{m}$ and $14 \mu \mathrm{m}$ in diameter, respectively. From these morphological characteristics, however, a specific identification cannot be made.

The results of the indirect immunofluorescence analyses are given in the Table. As noted, the amoebae in the brain sections stained only with the $A$. culbertsoni antisera. They failed to give positive fluorescence with the remaining acanthamoeba antisera or antisera produced against $N$. fowleri or
Table Results of indirect immunofluorescence antibody tests on brain tissue sections

\begin{tabular}{lll}
\hline & Dilution & Result \\
\hline Whole-cell antisera* & & \\
E. histolytica (HK-9) & $1: 32$ & 0 \\
N. fowleri (ITMAP-359) & $1: 16$ & 0 \\
A. castellanii (Neff) & $1: 16$ & 0 \\
A. polyphaga (P-23) & $1: 16$ & 0 \\
A. rhysodes (CCAP-1534) & $1: 16$ & 0 \\
A. palestinensis (Reich) & $1: 16$ & 0 \\
A. astronyxis (Ray) & $1: 8$ & 0 \\
A. culbertsoni (A-1) & $1: 8$ & + \\
& $1: 16$ & 0 \\
Plasma membrane antisera & & \\
A. castellanii (Neff) & $1: 32$ & 0 \\
A. rhysodes (CCAP-1534) & $1: 32$ & 0 \\
A. culbertsoni (A-1) & $1: 128$ & ++ \\
& $1: 256$ & + \\
& $1: 512$ & + \\
\hline
\end{tabular}

*The dilutions of the antisera used give an optimum staining of tissue sections and provide no cross-reactions except between $A$. castellanii, $A$. polyphaga, and $A$. rhysodes (see Material and methods).

E. histolytica. With anti- $A$. culbertsoni whole-cell sera, the amoebae in the tissue fluoresced only at a low titre $(1: 8)$, whereas with antisera raised against purified plasma membranes of $A$. culbertsoni, the amoebae stained to the homologous titre $(1: 512)$. The amoebae showed bright fluorescence $(2+$ response) (Table) to a dilution of 1:128 (Fig. 1), and a less bright fluorescence $(1+$ response) (Table) to a dilution of $1: 512$. At the high dilutions the fluorescence appeared mainly on the membranes of the cytoplasmic vacuoles in the amoebae (Fig. 2).

Immunofluorescence titres of $1: 2$ and $1: 4$ were obtained when the patient's serum was tested against $A$. castellanii and $A$. culbertsoni, respectively. The serum agglutinated $A$. culbertsoni trophozoites to a titre of $1: 32$ but did not agglutinate $A$. castellanii amoebae. No precipitin bands were evident after reaction of the patient's serum with soluble antigens of the type species of acanthamoeba.

\section{Discussion}

Approximately 15 cases of human brain infections caused by Acanthamoeba have occurred during the last decade (Willaert, 1974; Bhagwandeen et al., 1975; Ringsted et al., 1976; Willaert and Stevens, 1976; Martinez et al., 1977; Willaert, 1977; Willaert and Stevens, unpublished results of cases personally communicated by Healy and by Dr C. A. Garcia, Tulane University, New Orleans, Louisiana). In all the cases reported before 1976 the amoebae were diagnosed only at the generic level from the presence of cysts and the morphological appearance of the trophozoites in tissue sections. The lack of specific antisera produced against Acanthamoeba species 


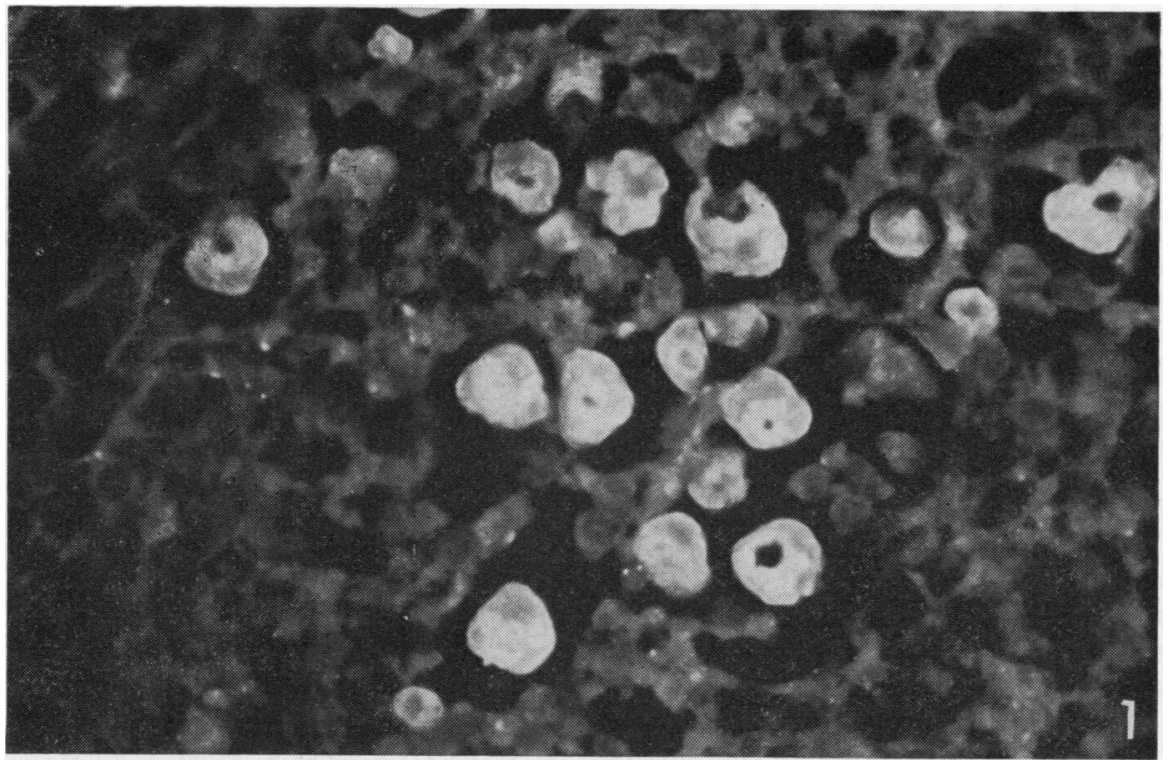

Fig. 1 Bright fluorescent staining of amoebae in the brain sections with anti-A. culbertsoni plasma membrane antiserum (dilution 1:32). $\times 750$.

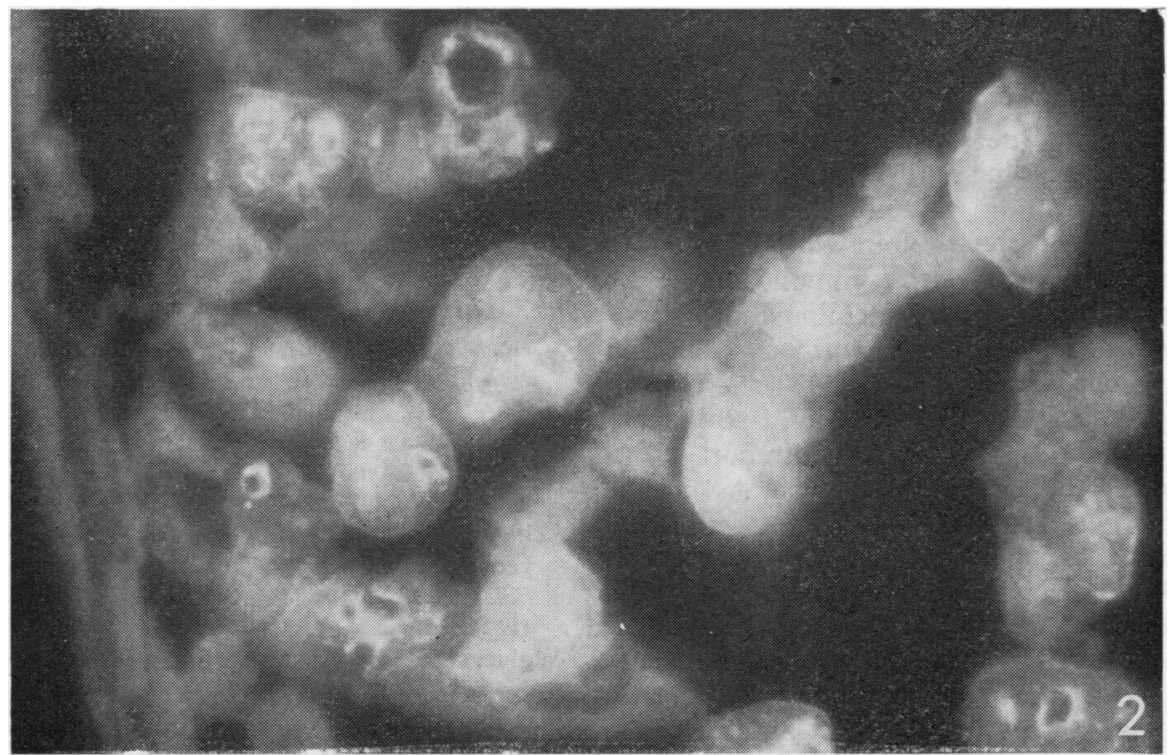

Fig. 2 Indirect immunofluorescent staining of brain sections with anti-A. culbertsoni plasma membrane antiserum (dilution 1:256). The fluorescence appears mainly on the membranes of the cytoplasmic vacuoles of the amoebae. $\times 1250$.

precluded identification of the infective species. For example, Jager and Stamm (1972) reported that the amoebae in the brain tissue sections reacted with four different antisera that had been produced against $A$. culbertsoni and $A$. castellanii and against two strains identified only as Ryan and $\mathrm{HN}-3$. These are prob- ably strains of $A$. castellanii. Jager and Stamm obtained the highest reaction with the Ryan antiserum (1:256), whereas $A$. culbertsoni antiserum gave positive fluorescence to a titre of $1: 128$. Fluorescence was observed with low dilutions of the other $A$. castellanii antisera. From their results, however, 
Jager and Stamm were not able to make a specific identification of the infective species.

With the use of antisera produced against purified plasma membranes of Acanthamoeba species (Stevens et al., 1977) and against whole, living cells (shorttime immunisation) (Willaert, 1976), we have been able to make specific identification in seven out of eight cases of Acanthamoeba infections that had been recently diagnosed or previously reported. Our success in identifying the species with these sera strongly suggests that, like other organisms, the most immunogenic determinants of acanthamoeba are surface-associated.

In the present study our results indicated that the secondary infection in the patient was caused by $A$. culbertsoni. Antisera produced against whole living cells of seven different species did not react with the amoebae in the sections, whereas the antisera raised against whole cells and against purified plasma membranes of $A$. culbertsoni reacted positively. However, an important difference in titres was observed between the two antisera. The plasma membrane antiserum reacted with the amoebae in the sections to the homologous end titre. In contrast, the amoebae reacted with the whole cell antiserum to a titre of only 1:8 (homologous end titre 1:64).

The detection of agglutinating antibodies against $A$. culbertsoni in the patient's serum provided additional evidence for the specific identification. The low titre of agglutinating antibodies and the lack of detectable precipitating or fluorescing antibodies may be due to the depressed ability of the patient to elicit humoral antibodies during immunosuppressive treatment. Long storage and manipulation of the serum may also be a reason for antibody deterioration.

The identification of $A$. culbertsoni as the aetiological agent in this case is the second report of this species causing infection in humans. As in the present study, we used highly specific plasma membrane antiserum to make the diagnosis in the first case, which originated in Venezuela (Willaert and Stevens, 1976).

Since the awareness that Acanthamoeba species are able to produce infections in humans more cases have been diagnosed in the past two years than in the past decade. It is important to point out that six out of the eight recent cases occurred in immunosuppressed patients. Thus, physicians should seriously consider the possibility of invasion with Acanthamoeba species in secondary infections in the central nervous system of patients subjected to immunosuppressive therapy.

We thank Dr Val Jager, Veterans Administration
Hospital, Batavia, New York, for supplying the brain tissue and serum from the patient.

Part of this work was accomplished while one of us (EW) was on a NATO fellowship at the Medical Research Service (Dr A. R. Stevens' laboratory), Veterans Administration Hospital, Gainesville, Florida. Additional support for the work was from the Medical Research Service of the Veterans Administration.

\section{References}

Bhagwandeen, S. B., Carter, R. F., Naik, K. G., and Levitt, D. (1975). A case of hartmannellid amebic meningoencephalitis in Zambia. American Journal of Clinical Pathology, 63, 483-492.

Culbertson, C. G., Smith, J. W., Cohen, H. K., and Minner, J. R. (1959). Experimental infection of mice and monkeys by Acanthamoeba. American Journal of Pathology, 35, 185-187.

Jager, B. V., and Stamm, W. (1972). Brain abscesses caused by free-living amoeba probably of the genus Hartmannella in a patient with Hodgkin's disease. Lancet, 2, 1343-1345.

Martinez, A. J., Sotelo-Avila, C., Garcia-Tamayo, J., Morón, J. T., Willaert, E., and Stamm, W. P. (1977). Meningoencephalitis due to Acanthamoeba sp.: pathogenesis and clinico-pathological study. Acta Neuropathologica, 37, 183-191.

Ringsted, J., Jager, B. V., Suk, D., and Visvesvara, G. S. (1976). Probable Acanthamoeba meningoencephalitis in a Korean child. American Journal of Clinical Pathology, 66, 723-730.

Stevens, A. R., Kilpatrick, T., Willaert, E., and Capron, A. (1977). Serologic analyses of cell-surface antigens of Acanthamoeba spp. with plasma membrane antisera. Journal of Protozoology, 24, 316-324.

Willaert, E. (1974). Primary amoebic meningo-encephalitis. A selected bibliography and tabular survey of cases. Annales de la Société Belge de Médecine Tropicale, 54, 429-440.

Willaert, E. (1976). Etude immuno-taxonomique des genres Naegleria et Acanthamoeba (protozoa: Amoebida). Acta Zoologica et Pathologica Antverpiensia, 65, 1-239.

Willaert, E. (1977). Immunotaxonomy of the genera Naegleria and Acanthamoeba and its diagnostic consequences on cases of amoebic meningitis. Giornale di Malattie Infettive e Parassitarie, 29, 680-689.

Willaert, E., and Stevens, A. R. (1976). Indirect immunofluorescent identification of Acanthamoeba causing meningoencephalitis. Pathologie et Biologie, 24, 545-547.

Requests for reprints to: $\operatorname{Dr}$ E. Willaert, Medical Research Service (151), Veterans Administration Hospital, Gainesville, Florida 32602, USA. 